ARTICLE

\title{
Exploring spatial averaging of contamination in fomite microbial transfer models and implications for dose
}

\author{
Amanda M. Wilson ${ }^{1,2,3 凶}$ and Rachael M. Jones ${ }^{1,2}$ \\ (c) The Author(s), under exclusive licence to Springer Nature America, Inc. 2021
}

BACKGROUND: When modeling exposures from contact with fomites, there are many choices in defining the sizes of compartments representing environmental surfaces and hands, and the portions of compartments involved in contacts. These choices impact dose estimates, yet there is limited guidance for selection of these model parameters.

OBJECTIVE: The study objective was to explore methods for representing environmental surface and hand contact areas in exposure models and implications for estimated doses.

METHODS: A simple scenario was used: an individual using their hands to contact their face and two microbially contaminated environmental surfaces. Four models were developed to explore different compartmentalization strategies: (1) hands and environmental surfaces each represented by one compartment, (2) hands represented by two compartments (fingertips vs. nonfingertip areas) while environmental surfaces were represented by one compartment, (3) hands represented by a single compartment and environmental surfaces represented by two compartments, and (4) hands and environmental surfaces each represented by two compartments. Sensitivity analyses were conducted to evaluate the influence of heterogeneous surface contact frequency, hand contact type, and hand dominance on dose.

RESULTS: Estimated doses were greatest when hand areas and environmental surfaces were each represented by two compartments, indicating that surface area "dilutes" contaminant concentration and decreases estimated dose.

SIGNIFICANCE: Model compartment designations for hands and environmental surfaces affect dose estimation, but more human behavior data are needed.

IMPACT STATEMENT: A common problem for exposure models describing exposures via hand-to-surface contacts occurs in the way that estimated contamination across human skin (usually hands) or across environmental surfaces is spatially averaged, as opposed to accounting for concentration changes across specific parts of the hand or individual surfaces. This can lead to the dilution of estimated contaminants and biases in estimated doses in risk assessments. The magnitude of these biases and implications for the accuracy in risk assessments are unknown. We quantify differences in dose for various strategies of compartmentalizing environmental surfaces and hands to inform guidance on future exposure model development.

Keywords: Fomite transmission; risk assessment; infectious disease modeling Journal of Exposure Science \& Environmental Epidemiology (2022) 32:759-766; https://doi.org/10.1038/s41370-021-00398-2

\section{INTRODUCTION}

Exposures through contacts with contaminated surfaces and subsequent touching of the face [1-3] or contact of contaminated objects with the mouth $[4,5]$ pose a health risk regarding many contaminants, including pesticides [6], heavy metals [7, 8], and microorganisms [1, 4]. Dermal absorption is an additional concern for some contaminants, such as pesticides [9]. Models that characterize exposures to contaminants through contact with environmental surfaces require parameters describing the parts and areas of hands and surfaces involved in the hand-to-environmental surface contact process. For example, the Residential Stochastic Human Exposure and Dose Simulation Model for Pesticides (Residential-SHEDS) model, which estimates nondietary ingestion and dermal exposures to pesticides among children, requires inputs of the surface area contacted and the frequency and duration of these contacts, among other variables [9]. The Child-Specific Aggregate Cumulative Human Exposure and Dose (CACHED) model, which estimates aggregate dose via dermal, inhalation, and dietary and non-dietary ingestion exposures, also requires hand surface area, surface area of contacts, and contact frequency [10]. These same parameters are required for

\footnotetext{
${ }^{1}$ Rocky Mountain Center for Occupational and Environmental Health, University of Utah, Salt Lake City, UT, USA. ${ }^{2}$ Department of Family and Preventive Medicine, School of Medicine, University of Utah, Salt Lake City, UT, USA. ${ }^{3}$ Department of Community, Environment \& Policy, Mel and Enid Zuckerman College of Public Health, University of Arizona, Tucson, AZ 85721, USA. ${ }^{凶}$ email: amwilson2@arizona.edu
} 
models that estimate microbial exposures via the fomite route $[4,11-17]$. When the assumptions used to define these parameters do not align with real-world conditions, errors can be introduced into the model that affect exposure estimates. Unfortunately for the modeler, human behaviors related to fomite transmission are highly complex and variable [12, 18-21], and small changes in the hand position during a surface contact affects the location and surface area on the hand to which contaminant is transferred, as well as the amount of contaminant transferred.

While modelers are generally guided by experimental and observational data when defining model parameters, the extent of knowledge and natural variability provides modelers with considerable latitude in their decision making. To date, analyses of the impact of model assumptions and parameterization on exposures through contact transmission have generally been limited to standard sensitivity analyses that explore the influence of variation in parameters on the output, such as through correlation $[4,12,16,17]$. Less work has explored how alternative model design may influence the model output. The objective of this study is to explore the impact of assumptions about exposure model parameters describing contact processes on exposure, with a specific focus on the impact of the number of compartments used to represent surfaces and hands involved in contacts, and the area of contact. The scenario under study involves viral exposure through contact with contaminated fomites. While microbial fomite exposures have some parallels to chemical fomite exposures, it should be noted that microbial fomite exposure insights may not be generalizable to those of chemical exposures due to lack of a dermal absorption route and differences in source, where the fomite may serve as the source itself. However, in modeling either type of process, considerations to the compartmentalization of hands and fomites must be considered.

This work is motivated by a specific concern related to heterogeneity in contamination on surfaces, including hands, and the extent to which model assumptions about uniform contaminant concentrations on surfaces may bias exposure models, if any. To explain, the part of a hand used for a hand-to-environmental surface contact may depend upon the environmental surface type and the hand configuration used [18]. For example, a single fingertip may be involved in the contact. If this occurs, the contaminant has heterogeneous loading, with contamination occurring only on the fingertip involved in the contact. However, in modeled representations of these events, the contaminant is typically averaged over the entire area of the compartment (e.g., the entire hand), thereby "diluting" the contaminant concentration across the entire surface area. As a result, the contaminant mass transferred to the fingertip is averaged over the entire hand, underestimating the contaminant concentration on the fingertip. This dilution is amplified by subsequent contacts that involve fractions of the compartment. Conversely, sequential contacts may involve different portions of the hand-e.g., the fingertip then the palm-and though contaminant acquired during a fingertip contact would not transfer during a palm contact, the model will predict contaminant transfer owing to the spatial averaging of the contamination across the compartment. Spatial averaging of contamination across environmental surfaces can introduce similar issues. This phenomenon may be exacerbated by heterogenous contact patterns, variability in the contact area, and heterogeneity in contamination density across different environmental surfaces. This may lead to under- or over-estimation of doses.

Prior work has demonstrated that heterogeneous contaminant loading can occur on the hand, on the level of $1 \mathrm{~mm}^{2}$, and can affect modeled exposures as the result of different hand positions during contact and activity patterns [22]. However, the implications of this heterogeneity for modeled doses have not been extensively explored, the fine scale of heterogeneity described is computationally expensive, and such data are available only for limited exposure scenarios [22]. Quantification of potential errors due to spatial averaging in simpler model frameworks, such as those explored herein, is needed before more advanced methodologies with more granular tracking of hand or surface loading can be more broadly incorporated. Despite the potential for errors that spatial averaging in compartment models can contribute, the effects on estimated exposures and doses have not been extensively described in the literature. This has relevance to the COVID-19 pandemic and future pandemics and outbreaks, as microbial exposure models are increasingly used for understanding viral exposure mechanisms [15, 17, 23-25].

\section{METHODS}

\section{Scenario and modeling approach}

The exposure scenario involved an individual making hand contacts with environmental surfaces and their facial mucosal membranes over the course of 20 min, transferring an infectious virus contaminant. The scenario was modeled using a compartmental mass-balance approach implemented using a Markov chain and simulated using the Monte Carlo method: This approach has been previously used to model virus exposures through the fomite route $[15,26]$. The four general categories of compartments included: (1) environmental surfaces, (2) hands of the individual, (3) facial mucous membranes of the individual, and (4) loss owing to virus inactivation. Compartments in the first two categories were varied to obtain the study objective. Specifically (Fig. 1):

- Model A included a single compartment for surfaces (a small and a large surface combined) and a single compartment for hands (right and left combined).

- Model B included a single compartment for surfaces (as in model A) and two compartments for hands that represented the fingertips (right and left combined) and other parts of the hands (right and left combined), respectively.

- Model C included a single compartment for hands (as in model A) and two compartments for surfaces (one small and one large).

- Model D included two compartments for surfaces (one small and one large, as in model C) and two compartments for specific hand sections (as in model B).

In models $A$ and $C$, because few contacts involve the entire hand surface area, contacts were considered to involve a fraction of the hand surface area, and the number of viruses transferred to or remaining on the hand after a contact was assumed to be uniformly distributed over the hand surface area. In models B and D, the hand surface area was separated into two compartments: fingertips and non-fingertip areas of the hand (Table 1, Fig. 1). Similar to model $A$, however, contacts were considered to involve a fraction of the fingertip surface area and non-fingertip surface area, and the number of viruses transferred to or remaining on in each compartment after a contact was assumed to be uniformly distributed across the compartment area. In all models, contacts with facial mucous membranes were assumed to involve only a single fingertip (a fraction of the total fingertip area), consistent with prior work $[1,15,25]$. Despite the use of 1 or 2 compartments, the total area of the hands and environmental surfaces and the frequency of the contacts with surfaces and the face are consistent between the two models. However, the fractions of compartment areas involved in contacts were altered to account for the size and function of objects represented by the two compartments (e.g., fingertip and nonfingertip areas of the hand).

The transfer of virus between model compartments was described using first-order rate constants, and used to calculate one-step transition probabilities between compartments as required for a discrete time Markov chain $[15,26]$. Where available, data were used to define the firstorder rate constants (Table 1). Timestep and iteration combinations were inspected to select a timestep and iteration combination that resulted in stable mean estimated doses. A timestep of $0.001 \mathrm{~min}$ with 5000 iterations of the Monte Carlo simulation was found sufficient to obtain stable results. Input parameters and their distributions are described in more detail in the supplemental materials.

In model D, where the large and small environmental surface were represented as two compartments, it was assumed the small environmental surface would contribute $2 / 3$ of the total amount of environmental surface contacts while the large environmental surface would account for the other third. The rationale was small environmental surfaces, such as door handles, are likely contacted more frequently than larger environmental surfaces, such 


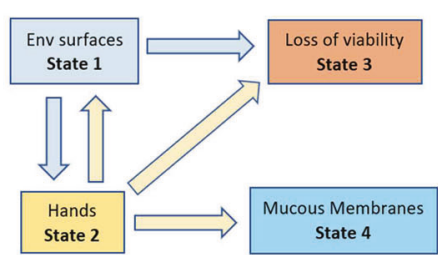

Model A

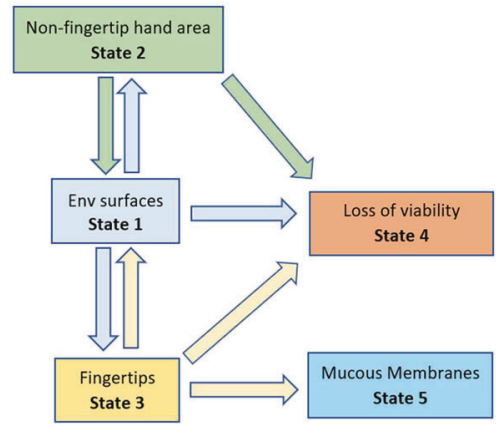

Model B

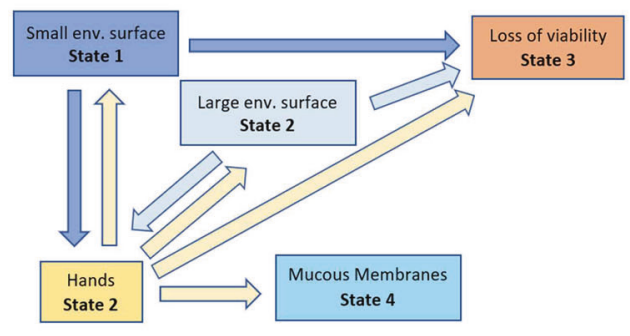

Model C

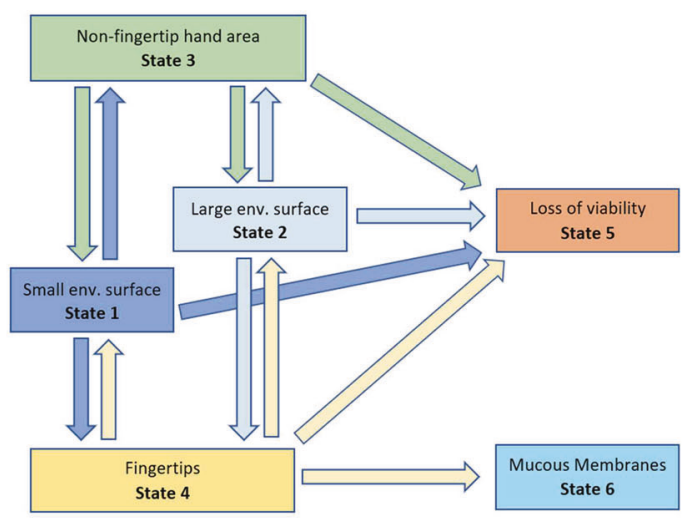

Model D

Fig. 1 Depictions of models A-D to explore dilution issues related to environmental surfaces and the hand.

as a desk. These surfaces (door handle and desk) were used to estimate environmental surface areas (Table 1, supplemental materials).

Fractional surface areas measured by AuYeung et al. were used to inform distributions describing the fraction of the hand or portion of the hand used for hand-to-environmental surface and hand-to-facial mucosal membrane contacts [18], while micro-activity data and other exposure modeling studies informed the frequency of these contacts $[2,14]$. It was assumed that the left or right hand would make contacts at the same rate [20]. However, hand dominance has been demonstrated in another study [27] and was therefore explored in the sensitivity analyses.

\section{Sensitivity analyses}

The effects of assumptions regarding the relative frequencies of hand-tolarge vs. hand-to-small environmental surface contacts and of fingertip-toenvironmental surface vs. non-fingertip-to-environmental surface contacts on estimated doses were investigated through modification of Model $D$, where there are separate compartments for the two fomites and separate compartments for the fingertip and non-fingertip areas of the hand (Table 2):

- Model D1 involved equal numbers of hand contacts with the small and large environmental surfaces, where $1 / 3$ of contacts were made with the fingertips and $2 / 3$ were made with the non-fingertip area.

- Model D2 involved equal numbers of hand contacts with the small and large environmental surfaces (as in Model D1), where $2 / 3$ of the contacts were made with the fingertip and $1 / 3$ were made with the non-fingertip area.

- Model D3 involved 2/3 of hand-to-environmental surface contacts being made with the large environmental surface and $1 / 3$ with the small environmental surface, where $1 / 3$ of contacts were made with the fingertips and $2 / 3$ were made with the non-fingertip area (as in Model D1).

- Model D4 involved 2/3 of hand-to-environmental surface contacts being with the large environmental surface and $1 / 3$ with the small environmental surface (as in Model D3), where $2 / 3$ of the contacts were made with the fingertip and $1 / 3$ were made with the nonfingertip area (as in Model D2).
Hand dominance many influence contact patterns [27], and this was explored by using states 3 and 4 in Model D (Fig. 1) to represent "hand 1" and "hand 2," respectively, rather than the fingertip and non-fingertip areas (Table 2). In these analyses, the total number of hand contacts with environmental surfaces was equal to that of the other models. Specifically,

- Model D5 involved equal numbers of contacts by hand 1 and hand 2 with environmental surfaces.

- Model D6 involved 10/90 split between hand 1 and hand 2 in contacts with environmental surfaces.

\section{RESULTS}

Having separate compartments for different hand areas and fomite areas resulted in the largest dose, where the mean estimated dose for model $D$ was 42.9 times, 25.5 times, and 2.7 times greater than those for models A, B, and C, respectively (Fig. 2 and S1A; Table S1). Between a single change in either separate compartments for hands or separate compartments for fomites, it appears that separate compartment for fomites had a larger influence on dose, where the mean estimated dose for model C (separate compartments for fomites, single compartment for non-fingertip and fingertip hand areas) was 16.1 times greater than model A (single compartment for non-fingertip and fingertip hand areas), single compartment for fomites, while the mean estimated dose for model B (single compartment for fomites, separate compartment for non-fingertip and fingertip hand areas) was 1.7 times greater than model A (Fig. 2 and S1A; Table S1).

When the environmental surfaces are treated as a single compartment (such as in models A and B), the starting concentration on the small environmental surface, which is assumed to be more contaminated, is diluted, as virus is "spread evenly" across the total small and large environmental surface area. To investigate the effect of the assumed contamination on the small environmental surface on 
Иัธ

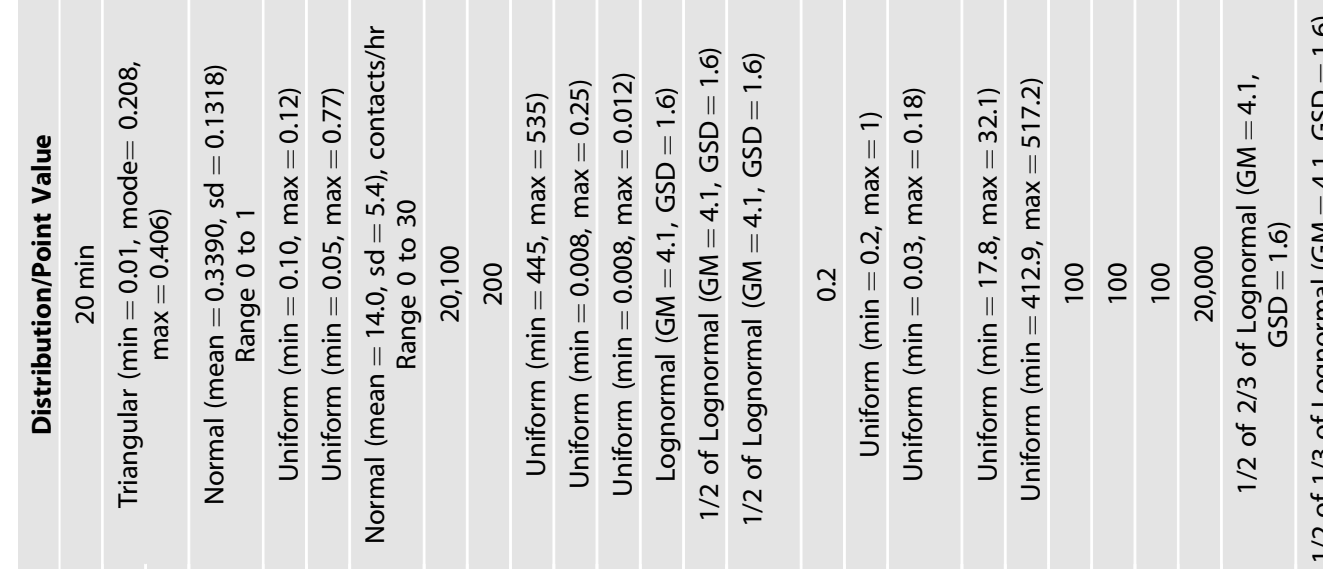

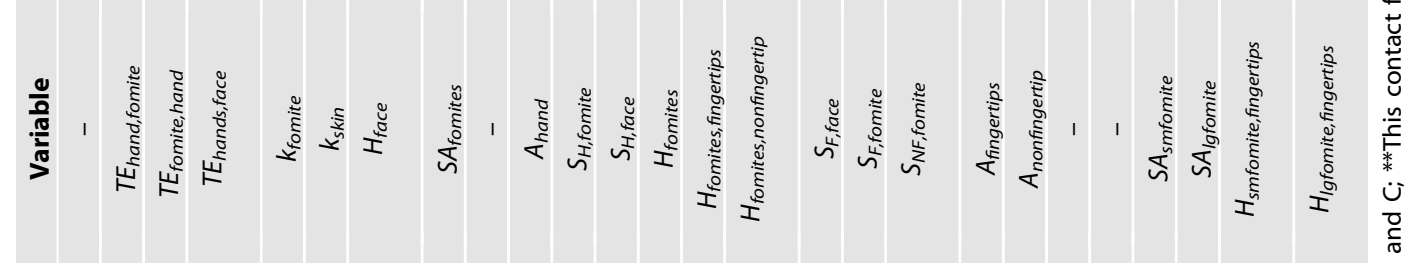

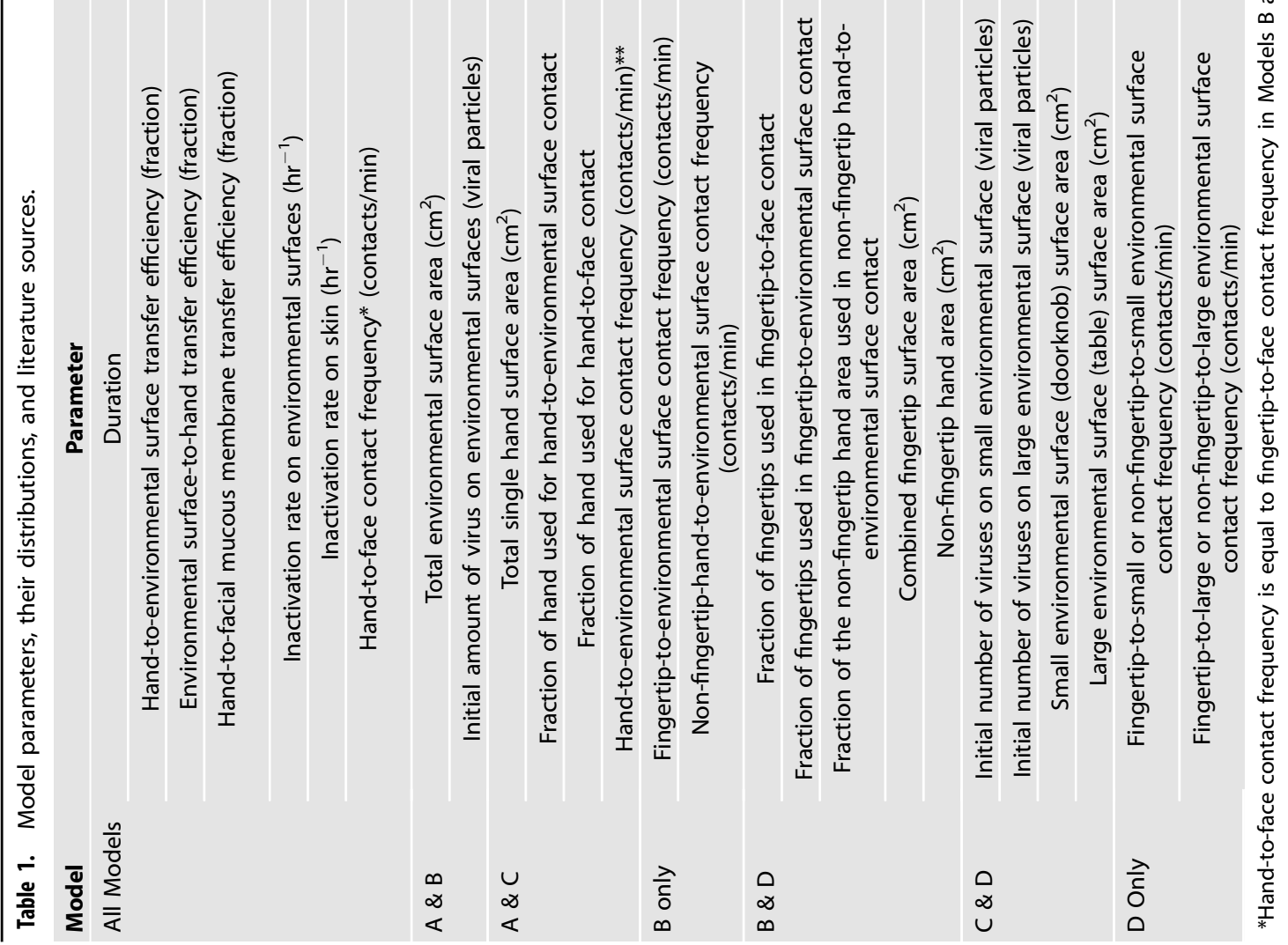


Table 2. Sensitivity analysis (SA) model descriptions ${ }^{a}$.

Contact frequency sensitivity analysis

Relative environmental surface contact frequency

Relative hand-area-specific contact frequency

Hand dominance sensitivity analysis

Fraction of total surface and face contacts
Fingertip: 1/3, Non-fingertip: $2 / 3$

Fingertip: $2 / 3$, Non-fingertip: $1 / 3$

Hand 1: 1/2

Hand 2: $1 / 2$

Hand 1: 1/10

Hand 2: 9/10
Large: $\mathbf{1 / 2}$, Small: $\mathbf{1 / 2}$

Model D1

Model D2

Large: 2/3, Small: 1/3

Model D3

Model D4

${ }^{a}$ For the sensitivity analysis exploring relative contact frequencies between hand areas and environmental surfaces, two relative environmental surface contact frequency scenarios were explored: (1) contact frequency for the large environmental surface was equal to contact frequency with the small environmental surface, and (2) contact frequency with the large environmental surface accounted for $2 / 3$ of environmental surface contacts while the small environmental surface accounted for the other third. Two relative hand-area-specific contact frequencies were explored: (1) the fingers were used for $1 / 3$ of environmental surface contacts and non-fingers were used for $2 / 3$, and (2) the fingers were used for $2 / 3$ of environmental surface contacts and non-fingers were used for $1 / 3$. The combinations of these scenarios were used to inform 4 sensitivity analysis models: models D1-D4. For comparison, in primary model D, the large fomite was touched $1 / 3$ of the time while the small fomite was touched $2 / 3$ of the time, and the fingertip and non-fingertip areas were used equally. Hand dominance was then explored with a modified version of Model D with separate compartments for each hand. A 50:50 and 10:90 split of one hand used over another were explored: models D5 and D6. For comparison, models A and C assume equal contact of fomites with the right and left hands via use of a single compartment for hands. Models B and D also assume equal contacts between the right and left fingertip or non-fingertip hand areas but are not comparable to models D5 and D6 due to the compartments being for fingertip or non-fingertip hand areas, specifically.

dose, model $D$ was simulated with a range of initial concentrations on the small environmental surface. When the initial concentration was smaller, smaller doses were observed (Fig. 3 ), which were similar to the mean doses estimated with models $A$ and $B$. When $50 \%$ of the viral particles were on the small environmental surface to start (1 viral particles $/ \mathrm{cm}^{2}$ on the small and 0.005 viral particles $/ \mathrm{cm}^{2}$ on the large environmental surface), larger doses, such as those for models $\mathrm{C}$ and D, were observed (Fig. 3), indicating that differences in dose for models $A$ and $B$ vs. models $C$ and $D$ were driven largely in part by assumed starting concentration on the surface.

Concentrations on the fingertip and non-fingertip areas increased when the small environmental surface had a separate compartment from the large environmental surface (Fig. S1B and D). This was likely a function of the small environmental surface being more contaminated in terms of density (viral particles $/ \mathrm{cm}^{2}$ ) than the large environmental surface at the start of the simulation (Table 1) and maintaining a relatively large concentration of virus over the simulation duration (Fig. S1D).

Models D1-D4 demonstrate the influence of assumptions regarding relative contact frequencies with heterogeneouslycontaminated environmental surfaces, and the frequency contacts with different parts of the hand on estimated doses (Fig. 4). Equal contact frequency with large and small environmental surfaces, as opposed to more contacts with the large environmental surface resulted in greater doses, where mean dose for models D1 and D2 were 1.3 to 1.2 times greater than that of models D3 and D4, respectively (Table S1). This is due to the small environmental surface having a larger concentration of virus than the large environmental surface in these models (Table 1). More frequent use of the fingertips resulted in greater doses, where models D2 and D4 resulted in mean doses that were 2.4 times and 2.7 times greater than models D1 and D3, respectively (Fig. 4, Table S1). This was a logical outcome, because the fingertips were used for hand-to-facial mucosal membrane contacts, and thus contributed directly to dose.

Models D5 and D6 demonstrate that assumptions regarding hand dominance can affect estimated doses, where heterogeneous use of hands may result in greater dose than when hands are used at an equal frequency (Fig. 4). This assumes, however, that both hands contact the facial mucosal membranes and that hand dominance equally applies to hand contact behaviors with the facial mucosal membranes and environmental surfaces.

\section{DISCUSSION \\ Key findings}

Decisions regarding compartmentalization of portions of the hands and for environmental surfaces influenced dose, where doses were greatest when there were separate compartments for the fingertips, non-fingertip areas, and the two separate environmental surfaces (Fig. 2; Table S1). The primary models indicate that separate compartments for fomites may have a larger impact on dose than separate compartments for fingertip and non-fingertip hand areas. The effect of the compartments on dose is also influenced by heterogeneity of contamination between environmental surfaces (Fig. 3), heterogeneity in contact frequencies between environmental surfaces, and the heterogeneity in frequency of contact with different parts of the hand (Fig. 4). This is especially true when there is more frequent contact with the more contaminated environmental surface and more frequent use of the portion of the hand used for hand-to-facial mucosal membrane contacts (fingertips in this study) (Table 2; Fig. 4).

\section{Heterogeneous environmental surface contacts \& contamination}

Heterogeneity in environmental surface contact frequencies has been demonstrated for children and adults. For example, Phan et al. reported that healthcare workers' contacts with the patient were more frequent than with surrounding surfaces (e.g., tray table, bed, chair, etc.), which had more similar contact frequencies [28]. In another study of healthcare workers, patient notes were touched more frequently than the blood pressure stand or telephone, for example [29]. Heterogeneous environmental surface contact frequencies have been observed outside of healthcare contexts, where Beamer et al. demonstrated differences in contact frequencies and/or contact durations among material types for children's micro-activities, where behaviors were also influenced by age and sex [20].

Differences in contact frequency have implications for heterogeneity in contamination. Adams et al. demonstrated a positive, 


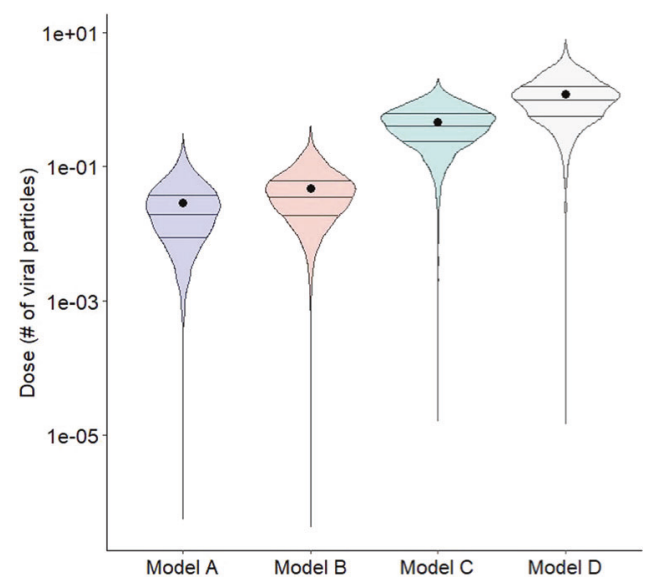

Fig. 2 Distributions of estimated doses for primary models A, B, C, and D. The 3 horizontal lines indicate the 25th, 50th, and 75th quantiles and the points indicate the means*. ${ }^{*}$ Doses are below 1 in some cases because these estimated doses represent an average of the dose over the simulation duration, taking into account variability and uncertainty in exposure-related parameters transitions of virus between compartments.

linear relationship between contact frequency and environmental surface contamination [30], implying that greater contacts with environmental surfaces may drive greater environmental surface contamination. This relationship supports the assumption in our model regarding a greater contact frequency with the more contaminated environmental surface, implying that separate compartments for environmental surfaces and consideration of heterogeneous contacts with environmental surfaces may be superior to using a single compartment and assuming a single hand-to-environmental surface contact rate. However, greater contact frequency does not always relate to greater environmental surface contamination, such as in cases where hand hygiene is conducted more regularly before certain environmental surface contacts than others [30]. More accessible surfaces that are touched more often may also be cleaned, sanitized, or disinfected more often. Because microbes transfer in both directions upon contact and according to a concentration gradient [31], a positive relationship between environmental surface contact frequency and environmental surface contamination implies that the hands are, on average, likely more contaminated than the environmental surface in contexts where that relationship holds, where hands are transferring virus to the surfaces.

While heterogeneity of contacts and contamination of environmental surfaces affects estimated dose and is important to consider in designing models and creating compartments, contact frequencies and environmental surface contamination are likely to be highly context specific. It may be challenging to determine how heterogeneous contact frequencies or contamination among environmental surfaces are expected to be, motivating the need to collect scenario-specific human behavior data to inform exposure and risk assessments.

\section{Hand configurations}

The representation of parts of the hand by multiple compartments has important implications for estimation of dose (Figs. 2 and 4), particularly when contacts with the point of exposure (e.g., facial mucous membranes) involve only a portion of the hand. However, as for many other model parameters, lack of data can affect implementation. For example, transfer of micro-organisms may vary across the hand owing to skin moisture and topography, contact angle, pressure, and average duration of contact, but studies of microbial transfer efficiency have focused only on fingertip contacts [31-35]. More data exist for chemicals, such as

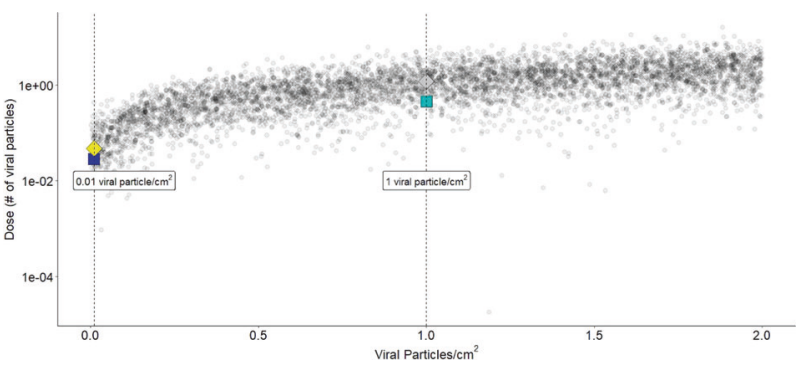

Fig. 3 Demonstration of how the compartment approach influences virus contamination on the small environmental surface, explaining, in part, differences in average dose across models. Results from primary models are shown (blue square $=$ model $A$, yellow diamond $=$ model $B$, turquoise square $=$ model $C$, gray diamond $=$ model $D$ ). This represents estimated doses using Model $D$, where the starting concentration on the small fomite is allowed to vary to demonstrate that differences in models $C$ and $D$ vs. models $A$ and $B$ are, in part, described by how the compartmentalization affects the assumed viral concentration on the small and more frequently contacted fomite. For models $C$ and $D$, virus contamination on the large environmental surface is 0.005 particles $/ \mathrm{cm}^{2}$ and 1 particle $/ \mathrm{cm}^{2}$ on the small surface. For models $\mathrm{A}$ and $\mathrm{B}$, virus concentration across both the small and large environmental surfaces is $~ 0.01$ particles/ $\mathrm{cm}^{2}$. This equates to the same number of viral particles per total surface area of fomites (200 viral particles $\left./ 20,100 \mathrm{~cm}^{2}\right)$. Doses are below 1 in some cases because these estimated doses represent an average of the dose over the simulation duration, considering variability and uncertainty in exposure-related parameters transitions of virus between compartments.

pesticide transfer during dry and wetted palm press contacts [36] and transfer of fluorescent markers during whole palm-side hand presses [37]. Another challenge for both microbial and chemical contexts is lack of data regarding what types of hand configurations are used for specific types of contacts. As the models in our study show, differences in frequencies of use for different portions of the hand can have effects on estimated doses (Fig. 4). In this study, it is assumed that fingertips are used for hand-to-face contacts, which does not capture moments where the side of a finger may be used to rub an eye, nose, or area of the mouth. To our knowledge, data describing specific parts of the finger used for hand-to-face contacts for adults are unavailable. Future data addressing this distinction could inform new compartment strategies, such as a "finger" compartment as opposed to "fingertip," or separate compartments for the fingertip and other areas of the finger that may be used for hand-to-face contacts.

\section{Hand dominance}

Hand dominance was found to impact dose estimates, where doses were greater when one hand was used more frequently than the other for all types of contacts (model D6) compared to equal use of both hands (model D5) (Fig. 4). While these results demonstrate the importance of accounting for hand dominance, there are limited data about the order in which right and left hand or dominant hand and non-dominant hand contacts are made, and results regarding the relative frequency of using one hand vs. another are inconsistent $[20,27]$. While the models in this study assumed that hand dominance applied to both environmental surfaces and hand-to-face contacts, Zhang et al. demonstrated that the non-dominant hand may be used more frequently for hand-to-face contacts in office settings [27]. It may be that preference or efficiency, rather than dominance, drives the contact pattern, at least in some scenarios [38]. Regardless, if the distribution of hand-to-face contacts between the hands differs from the distribution of hand-to-surface contacts, lower doses may result than those estimated by models that assume either equal use of the hands or more frequent hand-to-face contacts with the dominant hand, which would have a greater viral load 


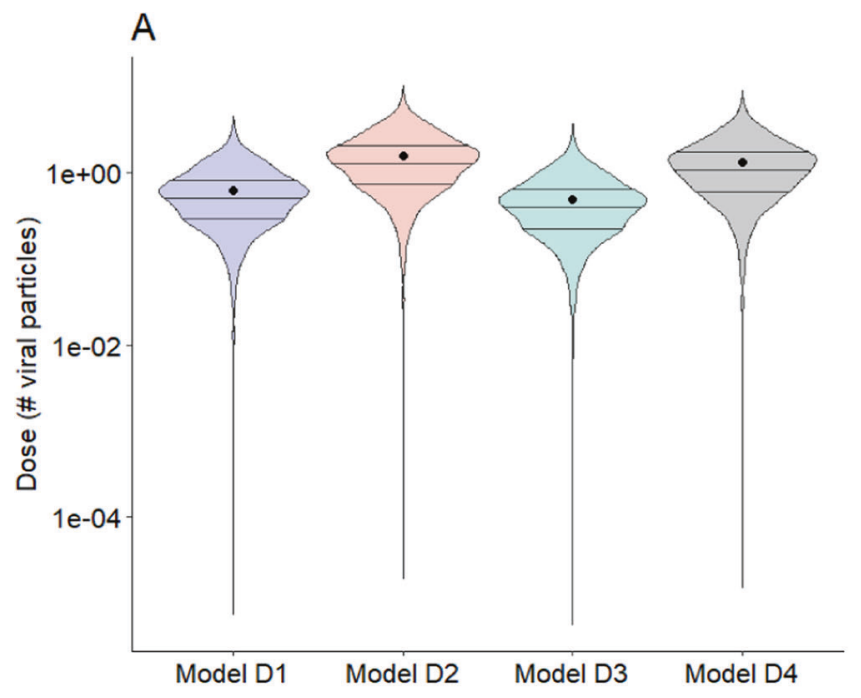

B

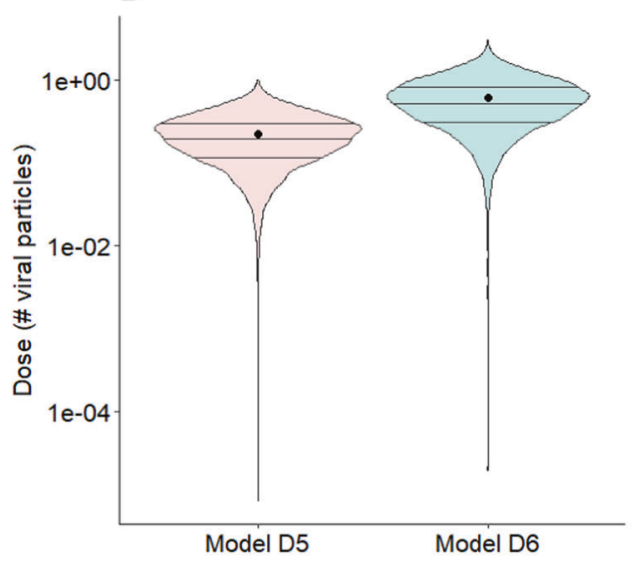

C

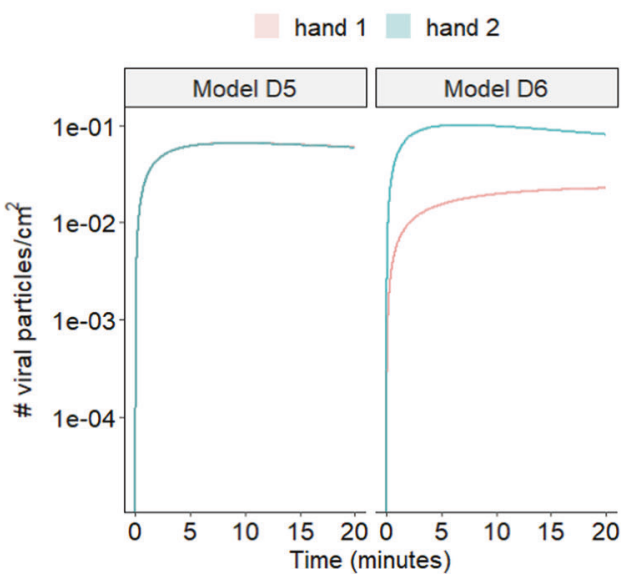

Fig. 4 Results from sensitivity analyses. A Estimated dose for sensitivity analysis models D1-D4, B Estimated dose for sensitivity analysis of hand dominance models D5-D6, C Concentration on hands 1 and 2 for sensitivity analysis models D5 and D6, where D5 results overlap for the two hands. In the violin plots, the 3 horizontal lines indicate the 25th, 50th, and 75th quantiles and the points indicate the means* ${ }^{*}$ Doses are below 1 in some cases because these estimated doses represent an average of the dose over the simulation duration, taking into account variability and uncertainty in exposure-related parameters and transitions of virus between compartments.

(Fig. 4). More data are needed to better describe heterogeneity in hand use, as more heterogeneous use of hands and parts of the hand may indicate greater bias in estimates of dose for models that do not account for hand dominance and preference (Fig. 4).

\section{CONCLUSIONS}

Based on differences in estimated doses, it is recommended that separate compartments are used for environmental surfaces and portions of the hand, especially when (1) heterogeneous contact frequencies and contamination levels with environmental surfaces are expected, (2) contact frequencies of different portions of the hand are expected, and (3) data are available to inform these microbial concentration and behavior parameters. An additional improvement would be to specify between right and left fingertip and non-fingertip areas of the hand to address hand preference and dominance in contacts. However, it may be difficult to anticipate the level of heterogeneity of contamination or contact frequencies and hand preference or dominance, because this may be highly task- or context-specific.

The findings of this study can be bolstered in future work with real-world data to validate the comparisons between models.
However, with or without real-world data, it should be considered which model is likely more representative of the exposure mechanisms at play. Future studies gathering temporal data describing frequency of contact with different fomites, what specific areas of fomites are touched, and concentration changes across individual fomites and hands would be useful for further elucidating these mechanisms and exploring more complex scenarios (more fomites, multiple individuals). In addition, mechanisms for chemical fomite-mediated exposure may differ, considering dermal absorption, differences in dynamics of transfer to fomites as opposed to from fomites relative to microbial transfer, and fomites serving as sources, in some cases. Future studies should explore how compartmentalization decisions in fomite modeling affect chemical dose and microbial dose estimates.

\section{REFERENCES}

1. Nicas M, Best D. A study quantifying the hand-to-face contact rate and its potential application to predicting respiratory tract infection. J Occup Environ Hyg. 2008;5:347-52.

2. Wilson $A M$, Verhougstraete $M P$, Beamer PI, King $M-F$, Reynolds $K A$ Gerba CP. Frequency of hand-to-head, -mouth, -eyes, and -nose contacts for 
adults and children during eating and non-eating macro-activities. J Expo Sci Environ Epidemiol. 2021:31:34-44.

3. Rahman J, Mumin J, Fakhruddin B. How frequently do we touch facial T-zone: a systematic review. Ann Glob Heal. 2020;86:75.

4. Julian TR, Canales RA, Leckie JO, Boehm AB. A model of exposure to rotavirus from nondietary ingestion iterated by simulated intermittent contacts. Risk Anal. 2009;29:617-32.

5. Xue J, Zartarian V, Moya J, Freeman N, Beamer P, Black K, et al. A meta-analysis of children's hand-to-mouth frequency data for estimating nondietary ingestion exposure. Risk Anal. 2007;27:411-20.

6. Hubal EAC, Suggs JC, Nishioka G, Ivancic WA. Characterizing residue transfer efficiencies using a fluorescent imaging technique. J Expo Anal Environ Epidemiol. 2005;15:261-70.

7. Sahmel J, Hsu El, Avens HJ, Beckett EM, Devlin KD. Estimation of hand-to-mouth transfer efficiency of lead. Ann Work Expo Heal. 2015;59:210-20.

8. Hauptman M, Bruccoleri R, Woolf AD. An update on childhood Lead poisoning. Clin Pediatr Emerg Med. 2018;18:181-92.

9. Zartarian VG, Ozkaynak H, Burke JM, Zufall MJ, Rigas ML, Furtaw E. A modeling framework for estimating children's residential exposure and dose to chlorpyrifos via dermal residue contact and nondietary ingestion. Environ Health Perspect. 2000;108:505-14.

10. Beamer PI, Canales RA, Ferguson AC, Leckie JO. Relative pesticide and exposure route contribution to aggregate and cumulative dose in young farmworker children. Int J Environ Res Public Health. 2012;9:73-96.

11. Ryan MO, Haas CN, Gurian PL, Gerba CP, Panzl BM, Rose JB. Application of quantitative microbial risk assessment for selection of microbial reduction targets for hard surface disinfectants. Am J Infect Control. 2014;42:1165-72.

12. Wilson AM, King M-F, López-García M, Clifton I, Proctor J, Reynolds KA, et al. Effects of patient room layout on viral accruement on healthcare workers' hands. Indoor Air. 2021;31:1657-72.

13. King $M-F$, Noakes $C J$, Sleigh PA. Modeling environmental contamination in hospital single- and four-bed rooms. Indoor Air. 2015;25:694-707.

14. Beamer PI, Plotkin KR, Gerba CP, Sifuentes LY, Koenig DW, Reynolds KA. Modeling of human viruses on hands and risk of infection in an office workplace using micro-activity data. J Occup Environ Hyg. 2015;12:266-75.

15. Jones RM. Relative contributions of transmission routes for COVID-19 among healthcare personnel providing patient care. J Occup Environ Hyg. 2020;17:408-15.

16. Kraay ANM, Hayashi MAL, Hernandez-Ceron N, Spicknall IH, Eisenberg MC, Meza $\mathrm{R}$, et al. Fomite-mediated transmission as a sufficient pathway: a comparative analysis across three viral pathogens. BMC Infect Dis. 2018;18:540.

17. Pitol AK, Julian TR. Community transmission of SARS-CoV-2 by surfaces: risks and risk reduction strategies. Environ Sci Technol Lett. 2021;8:263-269.

18. AuYeung W, Canales RA, Leckie JO. The fraction of total hand surface area involved in young children's outdoor hand-to-object contacts. Environ Res. 2008;108:294-9.

19. King M-F, Wilson AM, López-García M, Proctor J, Peckham DG, Clifton IJ, et al. Why is mock care not a good proxy for predicting hand contamination during patient care? J Hosp Infect. 2021;109:44-51.

20. Beamer PI, Luik CE, Canales RA, Leckie JO. Quantified outdoor micro-activity data for children aged 7 - 12-years old. J Expo Sci Environ Epidemiol. 2012;22:82-92.

21. Hubal EAC, Sheldon LS, Burke JM, McCurdy TR, Berry MR, Rigas ML, et al. Children's exposure assessment: A review of factors influencing children's exposure, and the data available to characterize and assess that exposure. Environ Health Perspect. 2000;108:475-86.

22. Canales RA The cumulative and aggregate simulation of exposure framework. Stanford University; 2004. PhD dissertation.

23. King M-F, Wilson AM, Weir MH, Lopez-Garcia M, Proctor J, Hiwar W, et al. Modelling the risk of SARS-CoV-2 infection through PPE doffing in a hospital environment. Indoor Air. 2021; Available from: https://doi.org/10.1111/ina.12938.

24. Harvey AP, Fuhrmeister ER, Cantrell M, Pitol AK, Swarthout JM, Powers JE, et al. Longitudinal monitoring of SARS-CoV-2 RNA on high-touch surfaces in a community setting. Environ Sci Technol Lett. 2021;8:168-175.

25. Wilson AM, Weir MH, Bloomfield SA, Scott EA, Reynolds KA. Modeling COVID-19 infection risks for a single hand-to-fomite scenario and potential risk reductions offered by surface disinfection. Am J Infect Control. 2021;49:846-848.

26. Weir MH, Shibata T, Masago Y, Cologgi DL, Rose JB. Effect of surface sampling and recovery of viruses and non-spore-forming bacteria on a quantitative microbial risk assessment model for fomites. Environ Sci Technol. 2016;50:5945-52.

27. Zhang N, Jia W, Wang P, King M-F, Chan P-T, Li Y. Most self-touches are with the nondominant hand. Sci Rep. 2020;10:10457.

28. Phan LT, Maita D, Mortiz DC, Bleasdale SC, Jones RM. Environmental contact and self-contact patterns of healthcare workers: Implications for infection prevention and control. Clin Infect Dis. 2019;69:S178-84.
29. Smith SJ, Young V, Robertson C, Dancer SJ. Where do hands go? An audit of sequential hand-touch events on a hospital ward. J Hosp Infect. 2012;80:206-11.

30. Adams CE, Smith J, Watson V, Robertson C, Dancer SJ. Examining the association between surface bioburden and frequently touched sites in intensive care. J Hosp Infect. 2017;95:76-80.

31. Wilson AM, King M-F, López-García M, Weir MH, Sexton JD, Canales RA, et al. Evaluating a transfer gradient assumption in a fomite-mediated microbial transmission model using an experimental and Bayesian approach. J R Soc Interface. 2020;17:20200121.

32. Lopez GU, Gerba CP, Tamimi AH, Kitajima M, Maxwell SL, Rose JB. Transfer efficiency of bacteria and viruses from porous and nonporous fomites to fingers under different relative humidity. Appl Environ Microbiol. 2013;79:5728-34.

33. Rusin P, Maxwell S, Gerba C. Comparative surface-to-hand and fingertip-tomouth transfer efficiency of gram-positive bacteria, gram-negative bacteria, and phage. J Appl Microbiol. 2002;93:585-92.

34. King M-F, López-García M, Atedoghu KP, Zhang N, Wilson AM, Weterings $M$, et al. Bacterial transfer to fingertips during sequential surface contacts with and without gloves. Indoor Air. 2020;30:993-1004.

35. Julian TR, Leckie JO, Boehm AB. Virus transfer between fingerpads and fomites. J Appl Microbiol. 2010;109:1868-74.

36. Clothier JM Dermal transfer efficiency of pesticides from new, vinyl sheet flooring to dry and wetted palms. 2000. Available from: https://nepis.epa.gov/Exe/ZyNET. exe/P100GN80.txt?ZyActionD $=$ ZyDocument\&Client $=$ EPA\&Index $=2000$ Thru2005\& Docs $=\&$ Query $=\% 28$ palm\%29ORFNAME\%3D\%22P100GN80.txt\%22ANDFNAME\% 3D\%22P100GN80.txt\%22\&Time $=\&$ EndTime $=\&$ SearchMethod $=1 \&$ TocRestrict $=$ n $\&$ Toc $=\&$ TocEntry $=\& Q$ Field $=\& Q F$. Accessed on June 24, 2021.

37. Hubal EAC, Nishioka MG, Ivancic WA, Morara M, Egeghy PP. Comparing surface residue transfer efficiencies to hands using polar and nonpolar fluorescent tracers. Environ Sci Technol. 2008;42:934-9.

38. Chatagny P, Badoud S, Kaeser M, Gindrat A-D, Savidan J, Fregosi M, et al. Distinction between hand dominance and hand preference in primates: a behavioral investigation of manual dexterity in nonhuman primates (macaques) and human subjects. Brain Behav. 2013;3:575-95.

39. van Doremalen N, Bushmaker T, Morris DH, Holbrook MG, Gamble A, Williamson BN, et al. Aerosol and surface stability of SARS-CoV-2 as compared with SARS-CoV-1. N Engl J Med. 2020;382:1564-7.

40. Harbourt DE, Haddow $A D$, Piper $A E$, Bloomfield $H$, Kearney $B J$, Fetterer $D$, et al. Modeling the stability of severe acute respiratory syndrome coronavirus 2 (SARSCoV- 2) on skin, currency, and clothing. PLoS Negl Trop Dis. 2020;14:e0008831.

41. U.S. Environmental Protection Agency. Exposure Factors Handbook 2011 Edition (EPA/600/R-09/052F) [Internet]. Washington, DC; 2011. Available from: https://cfpub. epa.gov/ncea/risk/recordisplay.cfm?deid=236252. Accessed on June 24, 2021.

\section{ACKNOWLEDGEMENTS}

Code is accessible via: https://github.com/awilson12/dilution_model_comparison. AM Wilson was supported by the Rocky Mountain Center for Occupational and Environmental Health (CDC/NIOSH T42/OH008414).

\section{AUTHOR CONTRIBUTIONS}

AW and RJ contributed to the intellectual development and writing of the manuscript. AW led coding development.

\section{COMPETING INTERESTS}

The authors declare no competing interests.

\section{ADDITIONAL INFORMATION}

Supplementary information The online version contains supplementary material available at https://doi.org/10.1038/s41370-021-00398-2.

Correspondence and requests for materials should be addressed to Amanda $M$. Wilson.

Reprints and permission information is available at http://www.nature.com/reprints

Publisher's note Springer Nature remains neutral with regard to jurisdictional claims in published maps and institutional affiliations. 